\title{
Comparison of Aero-Propulsive Performance Predictions for Distributed Propulsion Configurations
}

\author{
Nicholas K. Borer ${ }^{1}$, Joseph M. Derlaga ${ }^{2}$, Karen A. Deere ${ }^{3}$, Melissa B. Carter ${ }^{4}$, Sally A. Viken ${ }^{5}$, Michael D. \\ Patterson $^{6}$, Brandon L. Litherland ${ }^{7}$ \\ NASA Langley Research Center, Hampton, Virginia 23681 \\ Alex M. Stoll ${ }^{8}$ \\ Joby Aviation, Santa Cruz, California, 95060
}

\begin{abstract}
NASA's X-57 “Maxwell” flight demonstrator incorporates distributed electric propulsion technologies in a design that will achieve a significant reduction in energy used in cruise flight. A substantial portion of these energy savings come from beneficial aerodynamicpropulsion interaction. Previous research has shown the benefits of particular instantiations of distributed propulsion, such as the use of wingtip-mounted cruise propellers and leading edge high-lift propellers. However, these benefits have not been reduced to a generalized design or analysis approach suitable for large-scale design exploration. This paper discusses the rapid, "design-order" toolchains developed to investigate the large, complex tradespace of candidate geometries for the $X-57$. Due to the lack of an appropriate, rigorous set of validation data, the results of these tools were compared to three different computational flow solvers for selected wing and propulsion geometries. The comparisons were conducted using a common input geometry, but otherwise different input grids and, when appropriate, different flow assumptions to bound the comparisons. The results of these studies showed that the X-57 distributed propulsion wing should be able to meet the as-designed performance in cruise flight, while also meeting or exceeding targets for high-lift generation in low-speed flight.
\end{abstract}

\section{Introduction}

Electric propulsion technology has introduced or re-invigorated aircraft concepts that integrate aerodynamic and propulsive characteristics to yield a net performance benefit. These benefits include recapture of wing vortex energy [1,2], enhancing low-speed performance [3], acceleration and modification of airframe boundary layers [4,5], and many others. Due to the complex flowfields and interactions associated with these aero-propulsive integration technologies, it can be difficult to apply the benefits from a particular detailed study to a new aircraft design. This is because the traditional aircraft design process has viewed aerodynamic and propulsion system design and analysis for aircraft concepts as separable exercises that are matched during aircraft sizing and performance analysis [6,7]. To these traditional approaches, airframe-propulsion integration is viewed as a performance penalty, as it violates many of the assumptions used during the individual aerodynamic or propulsion analysis processes. As such, designorder tools or processes are necessary to enable successful, generic implementation of different integrated aerodynamic-propulsion concepts, much as dedicated design-order tools are used for different aerodynamic speed regimes or propulsion cycles today.

\footnotetext{
${ }^{1}$ Technical Lead/Aerospace Engineer, Aeronautics Systems Analysis Branch, MS 442, AIAA Senior Member.

${ }^{2}$ Aerospace Engineer, Computational Aerosciences Branch, MS 218, AIAA Member.

${ }^{3}$ Aerospace Engineer, Configuration Aerodynamics Branch, MS 499, AIAA Senior Member.

${ }^{4}$ Aerospace Engineer, Configuration Aerodynamics Branch, MS 499, AIAA Associate Fellow.

${ }^{5}$ Aerospace Engineer, Configuration Aerodynamics Branch, MS 499, AIAA Senior Member.

${ }^{6}$ Aerospace Engineer, Aeronautics Systems Analysis Branch, MS 442, AIAA Member.

${ }^{7}$ Aerospace Engineer, Aeronautics Systems Analysis Branch, MS 442.

${ }^{8}$ Aeronautical Engineer, 340 Woodpecker Ridge, AIAA Member.
} 


\section{A. Distributed Electric Propulsion for NASA's X-57 Flight Demonstrator}

Distributed propulsion is a class of integrated aerodynamic-propulsion concepts that does not have a formal definition, but generally involves distribution of the propulsion system beyond what may otherwise be most efficient from a traditional decoupled design standpoint, for the purpose of enhanced aircraft performance. Distributed propulsion may in fact contain a suite of integrated aerodynamic-propulsion concepts. NASA's X-57 "Maxwell" flight demonstrator [8], developed under the Scalable Convergent Electric Propulsion Technology Operations Research (SCEPTOR) project, will demonstrate distributed propulsion technologies integrated with electric motors, in a technology suite dubbed Distributed Electric Propulsion (DEP). The X-57 "Mod 4" variant, shown in Figure 1, leverages two different types of distributed propulsion concepts across 14 electric propulsors.

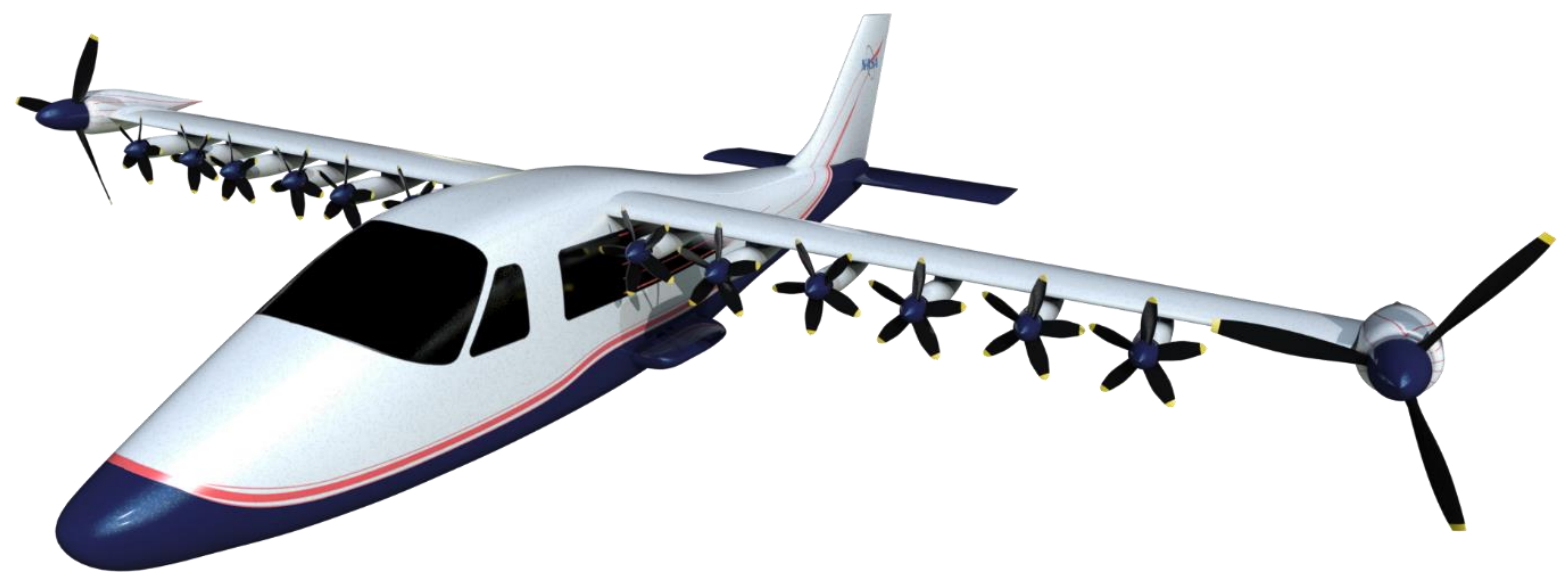

Figure 1: NASA's X-57 “Maxwell” Distributed Electric Propulsion Flight Demonstrator (Mod 4 Configuration)

Two large propulsors are used for the main propulsive power associated with all mission phases, with one propulsor located at each wingtip. These wingtip-mounted electric motors turn variable-pitch propellers that spin opposite to the wingtip vortex in flight, resulting in a performance increase that can be attributed to a reduction in induced drag, or a corresponding increase in propeller efficiency [9]. These are dubbed the "cruise propulsors." They will be present in the X-57 Mod 3 and Mod 4 variants [8].

The 12 remaining propulsors on the X-57 Mod 4 are distributed across the leading edge of the wing. These are smaller electric motors driving small-diameter, fixed-pitch, foldable propellers that are designed to operate in the low-speed range - takeoff, initial climb, approach, and landing. The primary purpose of these propellers is to augment the low-speed flowfield over the wing by enhancing dynamic pressure and changing the local angle of attack distribution $[10,11,12]$. This effectively scales the low-speed, high-lift capabilities of the aircraft, much like a large, complex flap system. As such, these are known as "high-lift propulsors."

\section{B. Sizing a DEP Aircraft}

The initial sizing for what would become the X-57 Mod 4 concept involved a wide tradespace exploration that considered over three million different discrete combinations of design variables [8]. Such a large exploration required a fast approach, with accurate trends with respect to the design variables of interest, in order to generate trades and sensitivities to guide the design selection. Unfortunately, the only approaches available to capture the integrated aero-propulsive benefits were (1) dated, empirical or quasi-theoretical methods, (2) new, sparsely validated design-order ${ }^{1}$ methods that integrate a variety of different design-order tools, or (3) detailed flow solvers (Navier-Stokes and otherwise) applied to the candidate geometries. The applicability of (1) was considered questionable for the expected flight regime and vehicle size investigated for SCEPTOR, so a number of tools related to (2) were developed or modified expressly for this task (this is not to say the dated methods were ignored, but were consulted and augmented where appropriate). The main issue with approach (3), the detailed flow studies, were twofold. First, they required far more geometric knowledge and detail than is applicable at early design stages [13]. The secondary issue was simply one of resources - the time (and computational facilities) needed to grid, set up, and

\footnotetext{
1 "Design-order" refers to tools that may sacrifice accuracy for generality and faster execution times, though such
} tools are expected to have accurate trends and compute reasonably accurate values within a known design domain. 
execute the complicated flowfields required for higher-fidelity computational or experimental studies, was simply prohibitive for large tradespace exploration efforts that involve more than a handful of candidate geometries.

This is not to say that the detailed flow solvers did not have their place in the design process. Rather, the tradespace developed from the design-order tools, combined with consultation with subject matter experts (particularly with respect to the disciplines not represented well in the design-order tools), helped to identify promising regions of the tradespace. This, in turn, generated designs that could be rendered in greater detail, allowing for investigation of more detailed solutions. The flow solvers could then be used on these preferred designs to check the validity of the design-order approach. For completeness, multiple different flow solvers and flow assumptions were used, in an effort to bound the uncertainty.

Given fewer limits on time or available resources, a more careful validation approach would certainly be reasonable. Controlled, detailed physical experimental investigations (such as large-scale powered wind tunnel models), coupled with high-fidelity computational flow solutions, can yield impressive and wide-ranging design rules for particular implementations of distributed propulsion. Unfortunately, the temporal and budgetary constraints associated with the execution of the SCEPTOR project (and related activities) have to date precluded the use of such approaches. Instead, SCEPTOR used a bootstrap-style approach of designing to a new method with limited validation, followed by adjustment and pseudo-validation by using multiple, different flow solvers and assumptions. This yielded a rapid, lower-cost mechanism to design a DEP flight demonstrator, with the acknowledged risk that the design may not perform as well as intended. From a design standpoint, pessimism, bounding, and margin were used in the final performance estimates prior to the handoff to more detailed analysis, fabrication, and integration.

This paper summarizes the design-order approaches used to design and analyze the integrated aerodynamicpropulsive performance of the X-57 flight demonstrator, the higher-fidelity flow solvers used for cross-evaluation and pseudo-validation ${ }^{1}$, and the results of this cross-comparison for selected studies associated with the SCEPTOR flight vehicle development. While the authors realize that any computational approach used to resolve fluid flow analysis can be referred to as Computational Fluid Dynamics (CFD), for this paper, CFD shall refer to higherfidelity computational analyses, namely, computational flow solutions to a form of the Navier-Stokes equations.

\section{SCEPTOR Design Tools for Distributed Electric Propulsion Concepts}

The design of the X-57 flight demonstrator required development of a number of design-order tools to help understand the salient trades amongst the different distributed propulsion technologies investigated for SCEPTOR. Rather than a "clean sheet" design, SCEPTOR chose to retrofit an existing light aircraft with a DEP wing [8] as a cost- and time-saving measure. This introduced explicit and implicit constraints, including limitations on gross weight, cruise speed, internal volume, balance, control power, and many more. Furthermore, the stepping-stone approach of the project, including the multiple configurations ("Mods") used during the build-up flight test approach, required that the eventual aircraft have adequate performance in each configuration, while meeting the program goals and constraints with the later configurations. For example, the cruise propulsion motors and propellers, as well as the baseline electrical system and interfaces, are essentially identical for the Mod 2, Mod 3, and Mod 4 configurations. The Mod 3 configuration first introduces the smaller, DEP-enabled wing with wingtipmounted propellers, so its performance needed to be adequate for takeoff and landing at higher speeds (given the lack of high-lift propulsors in the Mod 3 configuration), yet able to test the selected cruise point at cruise flight. Overall, these requirements separated the sizing and design-order tool analysis - one used to design a "cruise-sized" wing, assuming that high maximum lift was possible out of the high-lift system (flaps and high-lift propellers), and another used to size the high-lift propulsors and overall design constraints associated with low-speed performance.

\section{A. Cruise Drag Analysis}

The ultimate goal of SCEPTOR is to demonstrate how DEP can enable a dramatic reduction in onboard energy consumption through two major factors: (1) improvement in the efficiency associated with the transfer of onboard energy to thrust (motor efficiency), and (2) improvement in integrated aerodynamic-propulsive efficiency (higher effective lift-to-drag ratio). This combined metric was dubbed the "efficiency multiplier" - the ratio of the consumption of onboard stored energy for the baseline (unmodified) aircraft vs. the onboard energy consumed by the same aircraft with the DEP retrofit applied. The project selected a particular cruise point $-8,000$ feet altitude on a standard day at 150 knots true airspeed - as the benchmark for this efficiency metric [8]. Notably, the majority of the efficiency increase comes from the switch from gasoline piston engines in the baseline aircraft to high-efficiency

\footnotetext{
${ }^{1}$ The term "pseudo-validation" reflects the fact that the higher-fidelity flow solver toolchains used in this paper were not rigorously calibrated against known aerodynamic-propulsive test cases.
} 
electric motors, but a substantial increase still occurs due to the improvement in aerodynamic-propulsive efficiency. From the sizing perspective, the wing analysis for the cruise point needed to be able to capture the energy reduction benefit of operating wingtip-mounted propellers.

Previously, Miranda and Brennan developed a computational approach to estimate the effects of wingtipmounted propellers using a largely potential flow approach, including the development of a potential flow tool dubbed PROPWING [9]. This tool was intended to help with design and sizing of an aircraft with wingtip-mounted propellers. Unfortunately, during the rapid execution of the initial sizing for the SCEPTOR project, it was not possible to resurrect the PROPWING code in a manner to impact the sizing and analysis for the eventual X-57 aircraft. However, many of Miranda and Brennan's findings served to inspire the approach used to rapidly analyze wing-wingtip propeller design combinations.

Miranda and Brennan generalized Munk's Stagger Theorem to show that, for a constant spanload distribution and power input, the effective efficiency of a tractor installation (propeller mounted ahead of the wing) vs. a pusher installation (propeller mounted behind the wing) was identical. In the tractor configuration, the benefit was seen as a reduction in induced drag, whereas, in the pusher configuration, the benefit was seen as an equivalent increase in propulsive efficiency. Hence, while the X-57 design concept favored tractor installations for the wingtip propellers, sizing and performance analysis could consider the propeller located in either the tractor or pusher location and yield the same aerodynamic-propulsive benefit, at least from a potential flow perspective.

\section{Integrated Drag Analysis Approach}

Typical conceptual-level design approaches for drag analysis include a summation of drag components from various sources [14]. These build-up approaches are generally binned into lift-independent and lift-dependent sources, and may also include drag due to compressibility for high-speed aircraft. The tradespace exploration for the design of the DEP-enabled wing used a similar build-up approach. One key difference was the estimation of the interaction of wing lift distribution with the wingtip-mounted propeller, as this was needed to properly identify trends in cruise drag with various conceptual-level aircraft wing and propeller design parameters.

To accomplish this build-up, an integrated analysis approach was assembled and wrapped in a custom MATLAB ${ }^{\circledR}[15]$ environment. The components and information flow of this environment is shown in Figure 2. This involved the separate analysis of fuselage (black), nacelle (white), lifting surface induced and trim drag (blue), lifting surface parasite drag (green), and propeller (orange) contributions.

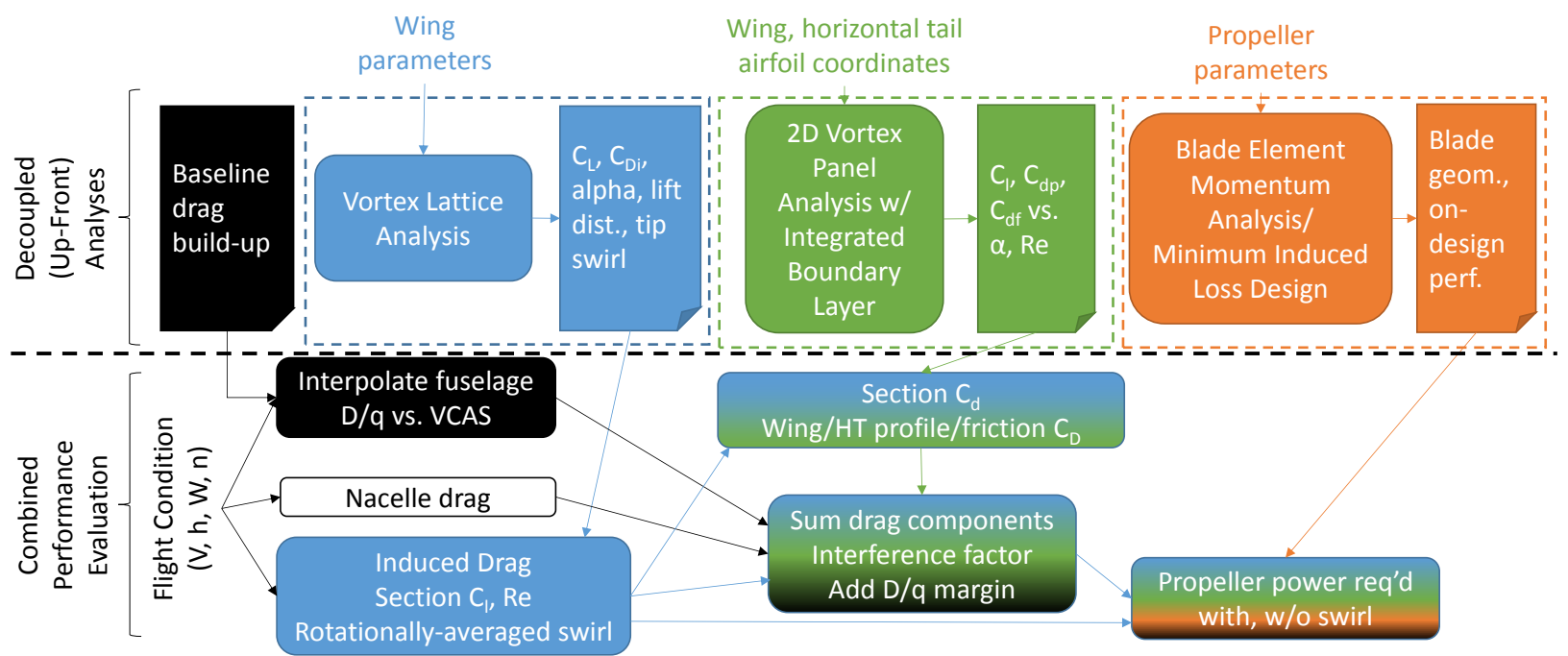

Figure 2: Design-Order Integrated Cruise Drag Analysis for SCEPTOR DEP Concepts

The baseline aircraft drag was estimated using spreadsheets developed for low-speed, general-aviation type aircraft created by Patterson et al. [16], calibrated to manufacturer-provided datasets and operating handbooks. This data was manipulated to give estimates of parasite drag for all aircraft components vs. calibrated airspeed (VCAS). The contribution of the baseline aircraft drag into the integrated cruise drag analysis was a table of the summation of fuselage and vertical tail $D / q$ vs. VCAS. The use of calibrated rather than true airspeed accounted for both Reynolds numbers effects and local flow angle of attack on the fuselage with changes in altitude and airspeed. Hence, for any given flight condition, the contribution of the fuselage and vertical tail was a simple interpolation from this lookup table based on the input calibrated airspeed. 
Nacelle parasite drag estimation for the wingtip cruise motors and high-lift motors came from a simple turbulent flat plate equivalent model multiplied by a form factor. The high-lift nacelles included a higher form factor to account for additional excrescence drag due to the folded propellers, as approximated by analysis conducted by one of the authors using CFD derived from the LEAPTech configuration [11]. Interference drag was estimated as 5\% of the sum of the parasitic drag components of the total aircraft. The lower number was selected such that margin was not stacked on top of margin - the main source of drag margin was an overall flat plate $D / q$ margin added to the vehicle. This created an independent variable that essentially allowed the designers to "dial in" the degree of pessimism by changing a single margin number.

\section{Lifting Surface Induced and Trim Drag}

Induced and trim drag was estimated with Athena Vortex Lattice (AVL), an open-source vortex lattice solver [17]. An AVL model of the wing and tail was generated from input design variables, executed locally, and parsed back into the integrated environment via a series of custom MATLAB ${ }^{\circledR}$ functions. This generated induced drag estimates, as well as trimmed angle of attack and stabilator positions for a sweep of input trimmed lift coefficients. Other important outputs included wing and horizontal tail lift distributions, which were used to help generate wing and tail parasite drag, as discussed in the Lifting Surface Parasite Drag discussion below.

The estimate of the effect of the wingtip propeller on vehicle performance leveraged Miranda and Brennan's assertion that the total overall induced drag and propulsive efficiency benefit was equal for tractor and pusher-type wingtip propeller configurations. It was impractical to modify AVL to include an input swirl distribution ahead of the wingtip from the propeller analysis, which is the physically correct case for the wingtip propeller concepts for SCEPTOR. Instead, the swirl was estimated from AVL and fed into the propeller analysis, as described under the Cruise Propeller Analysis discussion below. The swirl was estimated by placing a small "dummy wing" in AVL slightly behind the main wing element, as seen in Figure 3 (Left). Care was taken in auto-generation of the AVL geometries to ensure that no vortex filaments from the main wing were perfectly aligned with vortex filaments in the dummy wing, thus avoiding singularities in the solution. Using AVL input file commands, the dummy wing was exempted from the Kutta condition using the AVL NOWAKE command, and was excluded from summation into the wing and horizontal tail forces using the AVL NOLOAD command. The dummy wing was instead used to pull the induced angle of attack profile from the main wing, seen in Figure 3 (Right). This was then post-processed to generate a radially-averaged swirl component, which was eventually fed into the propulsion analysis. This knowingly did not capture all the vortex energy (namely, the viscous disruption of the vortex core), and was therefore a conservative estimate of the wingtip effect.

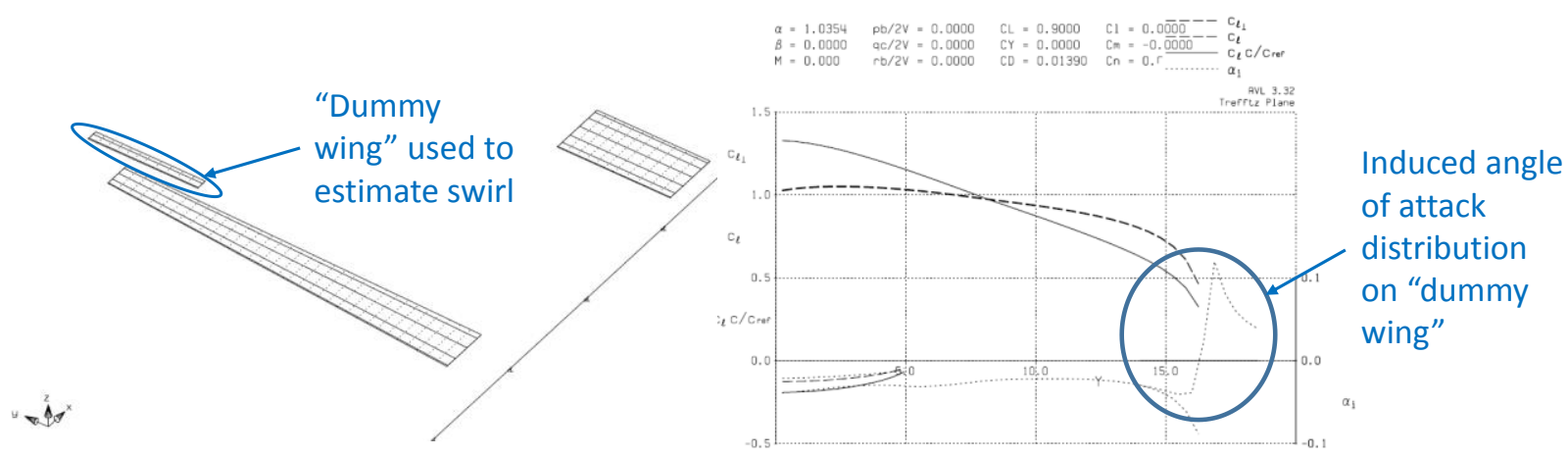

Figure 3: (Left) Vortex-Lattice Geometry for Induced and Trim Drag Calculation with "Dummy Wing" for Wingtip Propeller Effect Calculation; (Right) Induced Angle of Attack Distribution on "Dummy Wing"

\section{Lifting Surface Parasite Drag}

The parasite drag of the wing and horizontal tail was derived from numerical integration of two-dimensional airfoil analyses. The airfoils used for the project were considered fixed during the tradespace exploration. The horizontal tail airfoil was fixed by the retrofit aircraft (given the tail was unmodified from the baseline), whereas the wing airfoil was custom-designed for the SCEPTOR DEP application. Some manual iteration occurred between the project airfoil design during different tradespace exploration epochs; this is summarized in the SCEPTOR design paper [8]. For the integrated aerodynamic-propulsive analysis used for the design-order tools, the airfoils were simply seen as data tables of section lift and drag coefficients vs. angle of attack and Reynolds number.

The section data for the wing and horizontal tail airfoils were generated at various angles of attack and Reynolds numbers using XFOIL, an open-source two-dimensional panel solver with an integrated boundary layer model [18]. 
These included XFOIL estimates for transition from laminar to turbulent flow; this was necessary as the wing airfoil was designed as a laminar flow airfoil. Pessimism regarding early transition was instead captured via the flat-plate drag margin applied to the entire analysis.

Wing parasite drag was estimated via numerical integration over the lifting surfaces. First, the flight condition set the overall aircraft lift coefficient for the integrated analyses. The AVL-generated lift distributions for the wing and horizontal tails were found by interpolating this aircraft lift coefficient vs. the angle of attack sweep conducted during the AVL analysis. Then, for each section (20-50, depending on the surface), the section lift coefficient was interpolated from the corresponding AVL-generated lift distribution. The flight condition and local chord length were used to calculate the local Reynolds number. Together, this data was cross-referenced to the airfoil data tables, and interpolation was used to estimate the two-dimensional friction and profile drag of the corresponding lifting surface station. This section drag was then trapezoidally integrated over each lifting surface (wing and horizontal tail) to get an estimate of the total parasite drag contributions from each surface.

\section{Cruise Propeller Analysis}

The cruise propeller performance was estimated with XROTOR, an open-source Blade Element-Momentum Theory (BEMT) solver and Minimum Induced Loss (MIL) propeller design tool [19]. Initial tradespace exploration included a custom-designed MIL propeller; subsequent performance analyses of the selected design point across multiple flight conditions used a model derived from the selected commercially available propeller. In either case, the analysis remained the same. XROTOR allows users to input an upstream rotationally-averaged swirl velocity as a means to design co-axial rotors. This feature was used instead to analyze the effect of the wingtip-mounted propellers operating in the vicinity of the wingtip vortex. The rotationally-averaged angle of attack from the "dummy wing" in AVL was combined with the freestream velocity to generate a rotationally-averaged swirl input into the XROTOR model. This was compared to the same propeller without the swirl input as a means to estimate the total change in propulsive efficiency due to the presence of the input swirl. Per the Miranda and Brennan assertion, this change in propulsive efficiency was equal to the relative change in the total drag of the aircraft. This approach does not account for viscous effects such as vortex destruction due to counteracting shear of the interacting rotational flowfields, and also does not consider the effect of the increase in Reynolds number of the wingtip nacelle and outer portion of the wing by the accelerated propeller slipstream.

\section{B. High-Lift Propeller Design and Analysis}

The method used to design and analyze high-lift propellers was partially related to the high-lift goals associated with the overall aircraft design. For the LEAPTech experiment, the goal was to show a large increase in maximum lift coefficient, enabling a very small wing. Stoll et al. [11] discusses the method used for the design of the eventual LEAPTech wing and propeller system, which were largely optimized for low-speed performance.

As the LEAPTech experiment progressed, Borer et al. noted that current propeller design methods focused on setting chord and twist distributions that optimized propulsive efficiency, which effectively pushed for optimal thrust generation per unit input power [10]. However, high-lift propellers largely augment wing lift at low speed by scaling dynamic pressure, which implies that the method should maximize downstream velocity per unit input power. Borer and Moore hypothesized that the optimal thrust-producing designs may require a different approach to tailoring the downstream velocity distribution, and attempted a variety of arbitrary chord and twist distributions to discover design trends in high-lift propellers [20]. This showed, for high-lift propellers, that (1) airfoil design had little effect on on-design performance, but a large effect on off-design performance, and (2) wing-nacelle height offset interacted with velocity distribution and therefore total effective lift augmentation.

Other methods for analyzing the lift augmentation from high-lift propellers were also explored. A higher-order vortex lattice model, the Distributed Vorticity Element (DVE) method [21,22], was considered due to its ability to capture the mutual influence of the wing and propellers on one another [23]. Studies showed that the DVE method had promise for analyzing configurations with one or a relatively small number of propellers (including propellers placed at the wingtips, as was first demonstrated by Cole et al. [24]). As the number of propellers increased, the analysis time became burdensome to perform the desired types of design space explorations. The method was also not easily extensible to wings with flaps, though progress has been made on both of these fronts [25].

Subsequently, a simple model of lift augmentation from high-lift propellers based on fundamental "first principles" was developed by Patterson and German [12], and was later updated by Patterson et al. [26,27]. This model assumed the isolated propeller performance could be superimposed on the wing, and abstracted the propeller slipstream to a single, average axial velocity to determine lift augmentation. This lift augmentation model captured the impact of the propeller installation angle relative to the wing (i.e., the "flow turning" seen by the wing due to the 
propeller slipstream), as well as the velocity contribution. This also showed that the propeller installation angle was another important design parameter.

Another key component of the lift augmentation model developed by Patterson and German was the inclusion of the high-lift propeller diameter relative to the wing chord length. Ting et al. [28] showed that if the ratio of slipstream height to airfoil chord was less than approximately 1.0, the lift augmentation from an accelerated slipstream would be reduced significantly from the theoretical dynamic pressure ratio. The effect of finite slipstream height caused an increase in the power required to produce a specific desired lift augmentation when the propeller diameter was decreased relative to the local chord, which effectively imposed a lower bound on the high-lift propeller size. Finally, the distance of the propeller upstream of the wing was found to have significant impacts on the lift augmentation, principally due to propeller slipstream contraction. Although larger distances upstream could be beneficial to lift augmentation, other design considerations would likely lead to high-lift propellers being placed relatively close to the wing - approximately one propeller radius ahead of the leading edge of the wing [26]. Ultimately, this simple lift augmentation model was able to provide sufficiently accurate results for the lift augmentation, while requiring minimal computational resources.

\section{Blade Profile Design}

Additional research has focused on the design of high-lift propellers and the impact of the high-lift propeller design on the performance and design of the entire aircraft configuration. A high-lift propeller design method was introduced by Patterson et al. [26,29] that sought to maintain a near uniform axial velocity profile in the propeller slipstream to maximize lift augmentation. In order to produce blades with these near-uniform velocity profiles, the blade shapes differ from traditional MIL propellers. The high-lift propeller design method produces blades with increased chord lengths and twists near the root and decreased chord lengths and twists near the middle of the blade. These novel prop designs also tend to have slightly increased twist angles near the blade tip. Figure 4 shows a comparison of the axial velocity distributions, chord, and twist profiles between the high-lift propeller designs and the MIL counterparts, for the same average axial velocity (and hence otherwise equal lift augmentation potential).
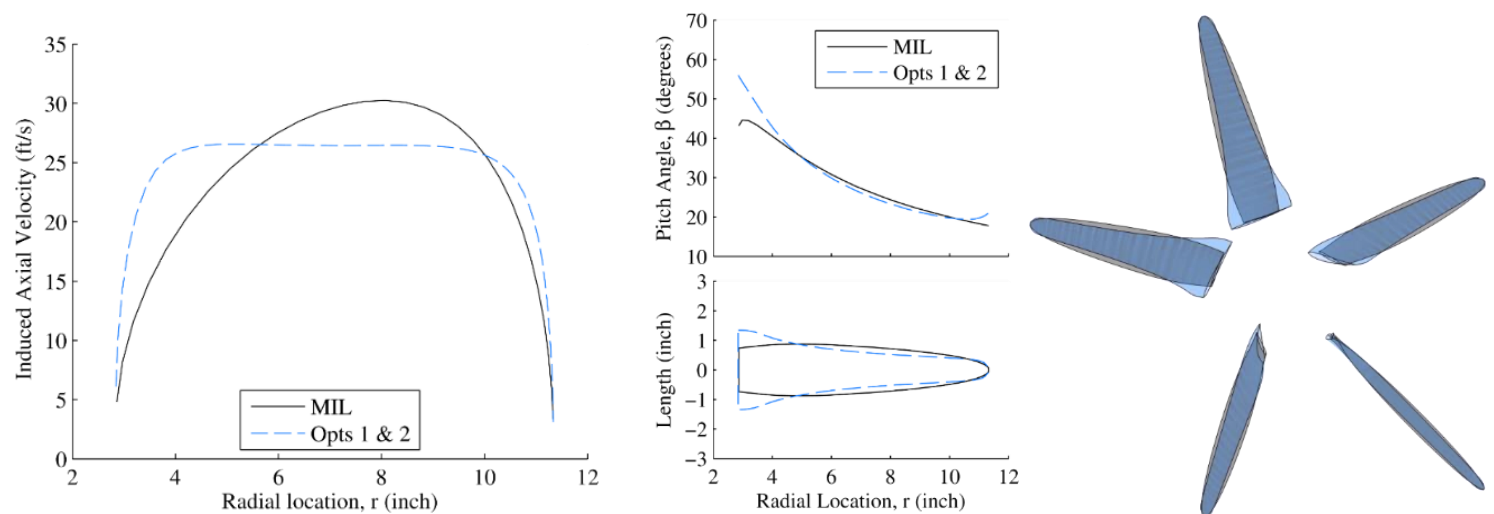

Figure 4: Comparison of High-Lift Propeller Designs (Blue, Dashed) vs. Minimum Induced Loss Designs (Black Solid) (Left) Axial Induced Velocity Distribution, (Center) Blade Twist and Chord Distributions, (Right) Isometric View

The impacts of high-lift propellers on the design and performance of the aircraft have been and continue to be studied. Trade studies analyzing different numbers of high-lift propellers for different SCEPTOR flight demonstrator configurations were performed, which illustrated the many, often non-intuitive tradeoffs that needed to occur [26,27]. These studies ultimately led to the selection of 12 high-lift propellers for the eventual X-57.

\section{Distributed Propulsion Pseudo-Validation Approach}

Multiple different CFD analyses, conducted over several different geometries, were used as part of SCEPTOR. Given the limited time and lack of relevant, high-quality validation data, the project adopted a strategy of using multiple teams with different high-fidelity flow solvers to focus on different problems. The CFD teams also conducted overlapping analyses as part of the pseudo-validation efforts. These were largely executed in parallel as the SCEPTOR design evolved; hence, at any given time, some of these teams could be analyzing slightly different geometries. If the CFD analyses largely agreed with each other, and better yet, with the design-order tools, the design was considered to be adequate for the project. Likewise, significant disagreement was cause to question the design choices made for the flight demonstrator. 
The pseudo-validation proceeded with a geometry build-up approach. Initially, the clean wing at cruise was evaluated with no nacelles or propellers. As the pseudo-validation progressed, nacelles, flaps, cruise propellers, and high-lift propellers were added for the different relevant flight conditions (cruise and stall). The different configurations were generally tracked by the components used in the geometry, as well as the specific revision number of the of the demonstrator concept. For this paper, the configurations use the following nomenclature:

- W: Wing

- $\quad C$ : Cruise (wingtip) nacelles

- $\quad H$ : High-lift nacelles

- $\quad F$ : Flap deployed

- $\quad P$ : Cruise propellers operating

- $\quad B$ : High-lift propellers operating

- \#.\#: Concept revision number

For example, WCP3.3 refers to analysis of the wing $(W)$, with the wingtip-mounted cruise nacelle $(C)$, with the cruise propeller operating $(P)$, of SCEPTOR's revision ${ }^{1} 3.3$ concept. Overall, the scope of the pseudo-validation build-up effort involved dozens of different permutations across three different CFD-based toolchains. For the purposes of brevity, this paper focuses on four different configurations, largely because they were used across each of the CFD tools (for similar or the same geometries), and represent good comparisons to the adequacy of the design-order tools. These configurations, and the associated analysis conditions, appear in Table 1. All references to "altitude" are used to identify associated atmospheric conditions per the corresponding altitude in the $1976 \mathrm{U}$. S. Standard Atmosphere.

Table 1: Configurations Used for Pseudo-Validation

\begin{tabular}{|c|c|c|c|c|}
\hline $\begin{array}{l}\text { Model (typical, } \\
\text { differs based } \\
\text { on config. ID) }\end{array}$ & & & & \\
\hline $\begin{array}{l}\text { Configuration } \\
\text { short name }\end{array}$ & $\begin{array}{l}\text { Cruise Wing, } \\
\text { Unpowered }\end{array}$ & $\begin{array}{l}\text { Cruise Wing, } \\
\text { Powered }\end{array}$ & $\begin{array}{l}\text { High-Lift Wing, } \\
\text { Unblown }\end{array}$ & $\begin{array}{l}\text { High-Lift Wing, } \\
\text { Blown }\end{array}$ \\
\hline $\begin{array}{l}\text { Config. IDs } \\
\text { (this paper) }\end{array}$ & WC3.3, WC4.1 & WCP3.3, WCP4.1 & $\begin{array}{l}\text { WF4.0, WCF4.0, } \\
\text { WCF4.1 }\end{array}$ & $\begin{array}{l}\text { WFB4.1, WFHB4.1, } \\
\text { WCFHB4.1 }\end{array}$ \\
\hline Altitude, $\mathrm{ft}$ & 8,000 & 8,000 & 0 & 0 \\
\hline Airspeed, & 150 & 150 & 58 & 58 \\
\hline
\end{tabular}

The geometries for each of these configurations were generated with OpenVSP [13,30], an open-source rapid aircraft geometry modeling tool. Each of the different CFD analyses required a slightly different path to move from OpenVSP to an appropriate grid geometry, which itself was not a trivial exercise. However, the ability to rapidly generate and grid multiple different permutations between multiple tools was a powerful capability. The complexities of the specific grid manipulations are well outside the scope of this paper, but the interested reader is encouraged to explore OpenVSP's documentation, as well as a recent presentation that included generation of Cartesian overset grids [31].

\section{A. OVERFLOW}

Simulations on structured, overset grids were performed using the OVERFLOW [32] solver. Due to the use of overset grids, various build ups of the baseline wing of the X-57 were studied, including the addition of cruise and high lift nacelles. In addition, overset grids allowed for rigid body motion simulations of the cruise and high lift propellers in isolation and installed on the X-57 geometry.

\footnotetext{
${ }^{1}$ Note the subtle difference in nomenclature between "revision" and "Mod." Given that the final design has always focused on a full DEP implementation, all design efforts centered on the X-57 Mod 4 configuration (new, smaller wing with wingtip cruise propellers and high-lift leading edge propellers). Hence, "revision 3.3" or "revision 4.1" both refer to a potential design for the X-57 Mod 4 configuration. All other "Mods" of the X-57 are merely artifacts of the build-up process used to develop confidence in key technology areas for SCEPTOR.
} 


\section{Grid Generation}

Near-body meshes were generated using the Chimera Grid Tools suite [33]; surface meshes were based on PLOT3D files directly exported from OpenVSP. The volume meshes were designed for a minimum viscous wall spacing requirement of $y^{+} \leq 1.0$, with five constant layers of growth off of viscous walls, and at least three layers of node overlap between adjoining grids. OVERFLOW-D mode was used (Domain connectivity function, or DCF), which enabled Cartesian off-body grids to be automatically generated by OVERFLOW extending approximately 100 chord lengths away from the wing.

\section{Computational Flow Solver and Solution Procedure}

A fifth-order WENO5M scheme [34] was used to perform variable reconstruction for the HLLE++ inviscid flux scheme [35], and a second-order central scheme was used for the viscous fluxes. Fully turbulent solutions were performed using a Spalart-Allmaras turbulence model, specifically SA-noft2-RC-QCR2000 (Rotation Correction plus Quadratic Constitutive Relationship) [36,37]. Results including transition were obtained using a k- $\omega$ SST-RCQCR2000 turbulence model [38,39] in conjunction with the Langtry-Menter CFX-v-1.1 transition model [40].

Euler implicit time marching was used to reach a steady-state solution for non-moving body simulations near cruise conditions. A second-order implicit time advancement method was used for moving body simulations, or near stall conditions. At least two orders of magnitude of convergence was desired per time step for unsteady simulations. For unsteady runs without propellers, at least 20,000 physical time steps were taken, given a time step based on the time required for a fluid particle to move $3 \mathrm{~cm}$. For propeller cases, an initial time step based on $1 / 4$ degree of rotation of the propeller was used, but this was relaxed to 1 degree of rotation per time step to speed convergence to a pseudo-steady state solution without undue change to force and torque predictions. For cases with installed high-lift propellers, four degrees per time step was used in order to quickly propagate the propeller wakes to the farfield. In general, at least 6 to 8 revolutions of a propeller were performed before time-averaging the results, and upwards of 16 were used for the installed, high-lift propeller cases.

\section{Convergence Criteria}

Besides the unsteady convergence requirements noted above, steady state forces were examined for standard deviations of less than 0.0001 . For steady simulations, forces and moments were typically averaged over the last 1,000 iterations. Unsteady simulations without propellers were averaged over the time to traverse one mean aerodynamic chord length, while simulations with propellers were averaged over the time to complete one revolution.

\section{B. STAR-CCM+}

STAR-CCM+ [41] is an unstructured cell-centered finite-volume-based solver. It was used for a variety of Reynolds-Averaged Navier-Stokes simulations, which included steady and unsteady modeling.

\section{Grid Generation}

The meshing model employed a template mesh constructed from hexahedral cells, from which it cut or trimmed the core mesh based on the starting input surface. The resulting mesh was composed predominantly of hexahedral cells with trimmed cells next to the surface. A robust automated prism layer meshing algorithm was used to capture the boundary layer, with wall $y^{+}$values kept in the 0.1 to 1.0 range. Mesh refinement was defined in regions such as the gap between the flap and the main element of the wing, around the propeller disks and streamtubes, and around wingtip vortices.

\section{Computational Flow Solver and Solution Procedure}

Most cases were run with steady-state calculations, although some wingtip propeller simulations employed implicit unsteady calculations, utilizing a time step size of $1^{\circ}$ of propeller rotation. Turbulence closure was achieved using the SST k- $\omega$ model [38,42], with transition modeled by the correlation-based $\gamma-\operatorname{Re}_{\theta}[40,43]$ model.

Typically, to decrease the computational expense of these simulations, instead of resolving the full geometry of the propeller, the propellers were modeled by prescribing uniform volume force distributions over cylindrical virtual disks. The volume forces varied in the radial direction, with the radial distribution of the force components based on the optimal Goldstein distribution, as described by Stern et al. [44]. The thrust and power were interpolated from experimental values of the uninstalled propeller in axial flow, with the implicit assumption that these values do not change substantially when the propellers are operated in front of the wing, and that the propeller normal force is negligible at the angles of attack of interest. Later analysis employed a similar model, with the assumed radial force distribution substituted with a model combining a blade-element method, with velocities calculated from local flow 
conditions. This analysis modeled the variation of thrust and normal force with angle of attack, and the azimuthal variation of thrust and torque.

\section{Convergence Criteria}

For steady-state simulations, the criteria used to monitor and determine solution convergence was based on the settling of parameters of interest, such as lift, pressure drag, and shear drag, for at least 300 iterations; however, this number of iterations varied with the type of simulation. Unsteady simulations were always periodic in nature (due to the propeller rotation), and in this case, the converge criteria comprised settling of residuals and propulsive efficiency within inner iterations, and periodic convergence of parameters such as lift, pressure drag, propeller torque, propulsive efficiency, etc. with time.

\section{FUN3D}

FUN3D [45] is a node-based, finite-volume discretization, Reynolds-averaged Navier-Stokes flow solver. It was used to evaluate angle-of-attack sweeps for the unpowered cruise wing configurations, as well as for the unpowered and powered high-lift wing configurations. The FUN3D simulations for this study used an actuator disc representation for the cruise and high-lift propellers, with thrust and power coefficient data input into the system (derived from the XROTOR blade element momentum analyses).

\section{Grid Generation}

An unstructured, mixed element mesh was used for the FUN3D simulations. The mixed element mesh had prisms in the boundary layer, tetrahedral cells in the far field, and pyramids to transition between the boundary layer and far-field cells. The semi-span mesh for the cruise wing with tip nacelles had 43.8 million mesh points. The semi-span mesh for the cruise wing with high-lift and tip nacelles had 47.8 million mesh points. The semi-span mesh for the high-lift wing with $30^{\circ}$ flap, with high-lift nacelles, with tip nacelles and also clustering in the regions of the propellers had 153 million mesh points. All meshes were designed for a $y^{+}=0.67$ and 34 layers within the boundary layer. Data from the cruise wing solutions and the high-lift wing without power show that $y+$ was less than 0.7 for all angles of attack. For the high-lift wing with high-lift power cases, the $y^{+}$varies up to 1.5 on the wing leading edge.

\section{Computational Flow Solver and Solution Procedure}

Low and moderate angle of attack cases were run with steady state calculations that used a local time step pseudo-time advancement scheme. The pseudo time advancement Courant-Friedrichs-Lewy (CFL) number was ramped up from 1 to 100 over 100 iterations for lower angles of attack, and the maximum CFL was limited to 40 for the higher angles of attack. At higher angles of attack, an optimized second-order backward differencing scheme was used for time-accurate temporal time integration. The Spalart-Allmaras turbulence model [46] was used with the rotation and curvature (RC) corrections, and the mean stress-strain Quadratic Constitutive Relationship (QCR) [37] turned on.

\section{Convergence Criteria}

The criteria used to monitor and determine solution convergence was a drop of at least two orders of magnitude for the flow solution residual, along with a standard deviation of less than 0.0012 for the lift coefficient $C_{L}$ and 0.0002 for the drag coefficient $C_{D}$, over 1000 iterations. For steady state solutions not meeting convergence criteria, the solutions were computed with time accurate CFD method.

\section{Design Validation Results}

The pseudo-validation cases (introduced earlier in Table 1) represented a sensible approach to ensure that the design-order tools, with their limiting assumptions, were capable of producing a design that could meet minimum requirements for success in the SCEPTOR project. The use of these tools with margins, bounding assumptions, and pessimism as prudent was intended to ensure that the performance of the eventual X-57 flight demonstrator will be adequate. In general, the individual design-order tools are well-characterized, industry standard tools, hence the pseudo-validation focused on how well they represented the integrated behavior of key DEP components.

\section{A. Cruise Wing, Unpowered}

The cruise wing was a logical initial choice for validation. The SCEPTOR project endeavors to show a significant reduction in cruise energy consumption due to aerodynamic-propulsive benefits, with one of the key 
benefits due to the use of a much smaller, higher-loaded wing at cruise. The smaller wing, enabled without sacrificing low-speed takeoff and landing performance due to the high-lift motors, pushes the range of maximum unpowered aerodynamic efficiency to higher speeds for a given flight altitude [8].

The initial pseudo-validation focused on the $W C$ (wing and wingtip cruise nacelle) configurations. At the time of the initial design studies, the details such as high-lift pylon fairing design and high-lift nacelle shape were reasonably immature - these details are seemingly small when trying to select the overall appropriate wing planform parameters, and the driving constraints (particularly high-lift nacelle diameter, set by the motor diameter) were as of yet unknown and fixed at nominal values in the design-order tools. Yet, to CFD tools, these small details can result in local-scale phenomena that can, in aggregate, significantly affect performance. Hence, when conducting trades for the cruise wing, the high-lift nacelles were considered to be a largely fixed drag penalty, or otherwise handled with drag margin. As such, it was most appropriate to consider only the wing performance at high speed.

The wing lift and drag characteristics for the unpowered cruise wing are shown in Figure 5. Here, the model labeled "design" is the aggregate prediction of the wing design process (power-off) shown in Figure 2 earlier. The design tools performed well for this configuration. This was expected, as the main tools used for the design - a vortex lattice code for lifting surface effects, with integration of a two-dimensional vortex panel code for airfoil effects - work well for the expected design (high aspect ratio, unswept wing) and flight condition (low subsonic). The biggest difference is seen between the models assuming fully turbulent flow, and those assuming some transition from laminar to turbulent flow, which is largely manifested in the drag.
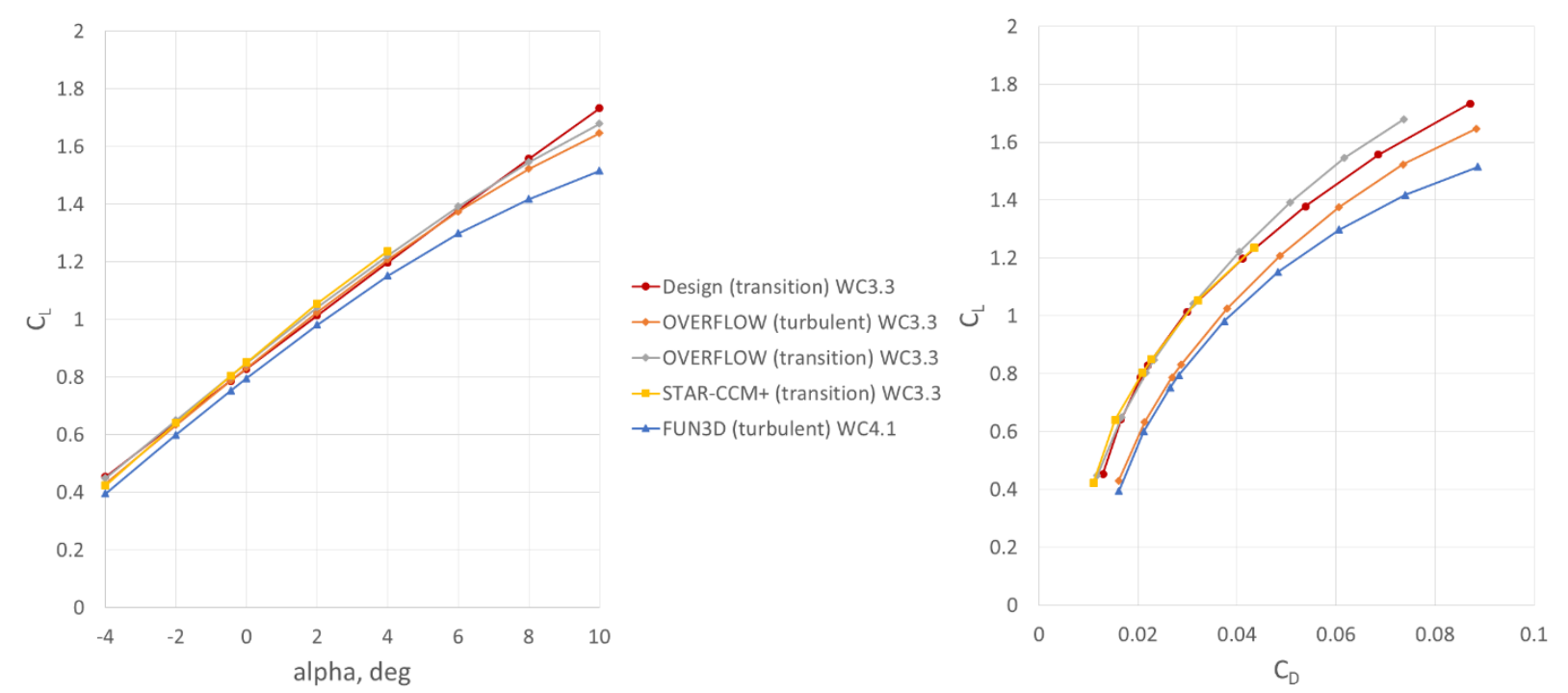

Figure 5: Comparison of Predicted Lift and Drag Performance for Unpowered Cruise Wing, No High-Lift Nacelles (WC)

There is also some apparent difference between the FUN3D and other codes at higher lift coefficients. However, one should note that the FUN3D runs in this plot are for the revision 4.1 geometry, whereas the other runs are given for the revision 3.3 geometry. The chief difference between revision 3.3 and revision 4.1 was a much longer wingtip cruise nacelle, as the 4.1 wing design occurred well after tradespace exploration, and when the details of the inner dimensional needs of the wingtip cruise nacelle (including motor, motor controller, data acquisition units, cooling flowpaths, and support structure) were determined. Overall, the diameter and length of the wingtip nacelle grew nearly $20 \%$ over this time. Later investigations of the high-lift configurations showed that the fairing for the 4.1 wing-wingtip junction was also inadequate, causing early separation at higher angles of attack. This largely explains the increasing offset seen from the FUN3D results to the others in terms of behavior at higher angles of attack. Still, such details could never be found with the design-order tools; hence, for the purposes of sizing a basic wing, the design-order tools appeared to be accurate, particularly near the cruise $C_{L}$ range (0.7-1.0).

\section{B. Cruise Wing, Powered}

The powered cruise wing cases proved to be more difficult to compare. This was due to a large number of variables and adjustments needed to ensure truth between the design-order tools and the CFD tools. For example, isolated unsteady rigid body simulation of the propellers in OVERFLOW revealed that a small, constant offset (order of $\sim 1$ degree) was necessary in the blade pitch axis to be able to match thrust levels reasonably. This could be 
due to a small offset in the zero-lift angle in the simple linearized airfoil model used in the XROTOR input. With this small offset, the thrusts matched and trended very well. However, the overall propulsive efficiency for the OVERFLOW runs also tended to be lower - this occurred due to everything from small issues with subscale discontinuities or waviness in the OpenVSP propeller model (the propeller models were far more sensitive than the wings in this regard), to larger issues such as the impact of nacelle blockage and other local flow phenomena that could not otherwise be accounted for in the XROTOR formulation. As such, trying to match the local cruise cases, which for the CFD meant running the as-created geometry vs. the somewhat ambiguous nature of the design-order tools, was indeed a challenge. The comparison of the results are shown in Figure 6 and Figure 7, for a region of lift coefficients and power settings that are in the vicinity of the steady-state cruise condition. For clarity, these figures include the original design-order unpowered and powered comparisons, as well as the unpowered and powered comparisons to individual CFD cases. The efficiency difference can be inferred by the relative difference between the unpowered (solid) and powered (dashed) cases in these figures.

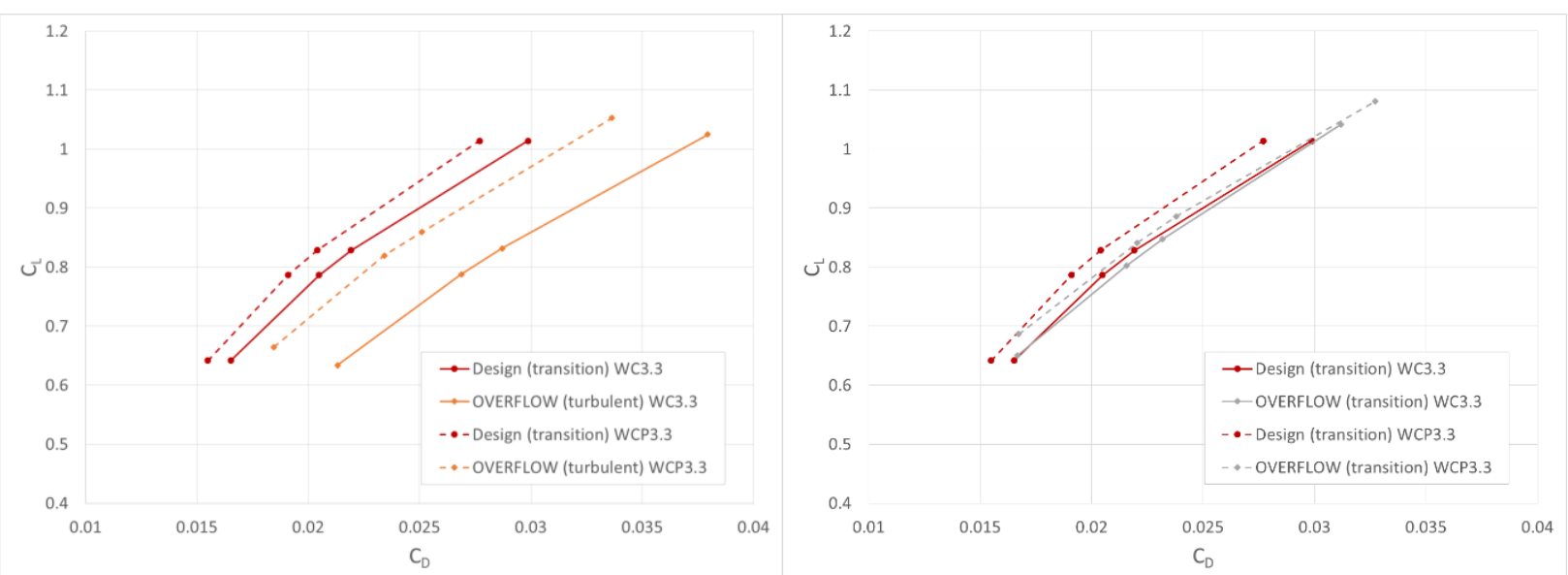

Figure 6: Drag Polar Comparison of Power-On (WCP) and Power-Off (WC) Wings - OVERFLOW with Fully Turbulent Boundary Layer (Left) and OVERFLOW with Boundary Layer Transition Model (Right)

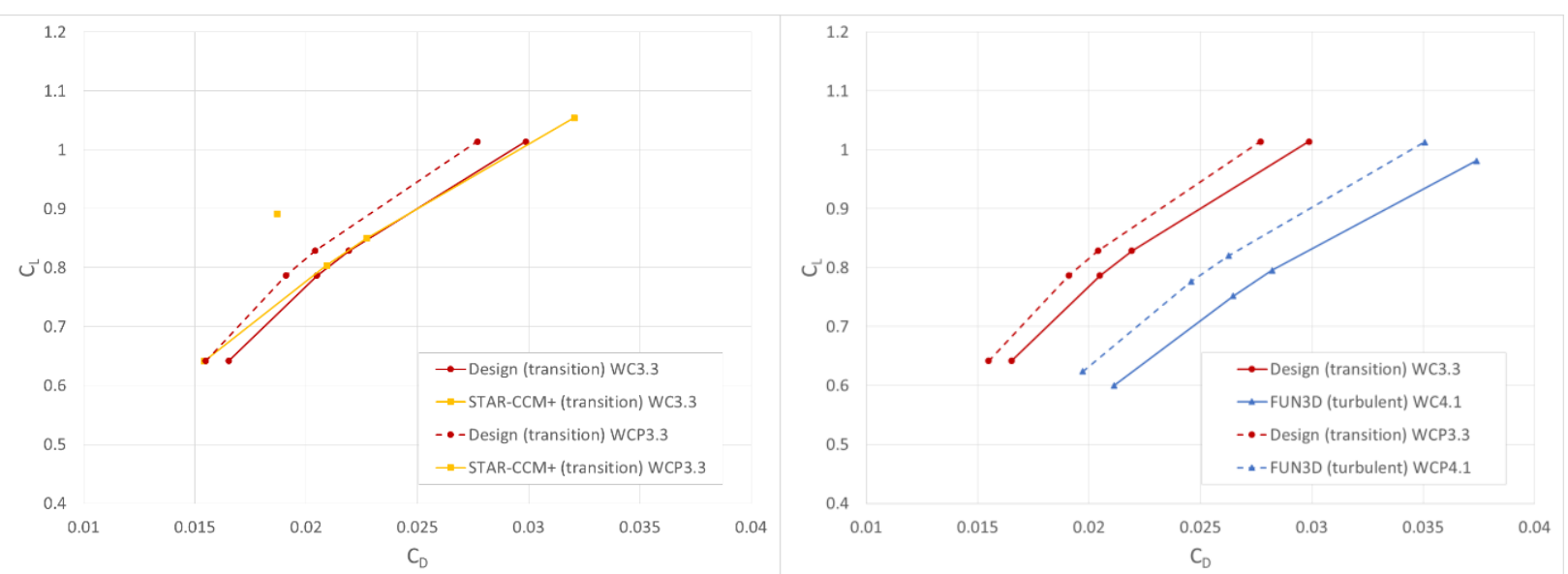

Figure 7: Drag Polar Comparison of Power-On $(W C P)$ and Power-Off $(W C)$ Wings - STAR-CCM+ with Boundary Layer Transition Model (Left) and FUN3D with Fully Turbulent Boundary Layer (Right)

Note that, per the discussion related to Figure 2, the benefit for the design-order cases appears as a change in total propulsive efficiency. Since the thrust at cruise is related to total drag, and not just wing drag, the estimated change in wing drag was found as follows:

$$
C_{D_{W C P}}=C_{D_{W C}}-\left(1-\frac{\eta_{\text {installed }}}{\eta_{\text {isolated }}}\right) C_{D_{\text {tot }}}
$$


where $C_{D_{W C P}}$ is the drag coefficient of the powered cruise wing (dotted red line in Figure 6 and Figure 7), $C_{D_{W C}}$ is the drag coefficient of the wing unpowered (solid red line in Figure 6 and Figure 7), $\eta_{\text {installed }}$ is the XROTORestimated propeller efficiency with the AVL-generated upstream swirl, $\eta_{\text {isolated }}$ is the XROTOR-estimated propeller efficiency in an isolated freestream, and $C_{D_{t o t}}$ is the total estimated drag of the aircraft at the specified angle of attack to maintain unaccelerated flight.

The difference between the unpowered and powered performance is about $7 \%$ of all wing drag for the designorder tools, $13 \%$ for the turbulent OVERFLOW model, $2 \%$ for the transitional OVERFLOW model, $18 \%$ for the single data point for the transitional STAR-CCM+ model, and $8 \%$ for the turbulent FUN3D model. This fairly wide range of benefit was likely due to the difficulties associated with matching all of the relevant information for the CFD models vs. the intent of the design-order models (particularly for the OVERFLOW cases, which needed actual blade geometries, rather than actuator discs). By comparison, Patterson and Bartlett's wind tunnel tests showed a $\sim 10 \%$ reduction in wing drag at lower lift coefficients and higher speeds [2]. Extrapolating results from Miranda and Brennan indicates drag reductions of $10-15 \%$ for this configuration [9]. Hence, other than the transitional OVERFLOW results, the design-order approach appears to be mildly conservative. Still, some caution remains related to the OVERFLOW results. There may be localized flow phenomena that are captured by the rigid body motion analysis, which are not otherwise realized by the design-order tools or CFD tools that used actuator discs.

\section{High-Lift Wing}

The unblown and blown high-lift cases involved a substantial amount of effort, and took place months after the initial design-order tool pseudo-validation. Hence, the comparisons in this section all refer to the revision 4.0 or later versions of the aircraft geometry, which was the first geometry release with details of the custom, single-hinge point Fowler flap that extends throughout most of the span of the X-57 Mod 3 and Mod 4 wing. The bulk of the unblown high-lift wing results occurred prior to better definition of the high-lift nacelles; hence, the unblown cases with highlift nacelles were not performed by a plurality of the CFD codes. This paper only compares unblown high-lift wing results without the high-lift nacelles, largely to show the difference in lift augmentation from the unblown high-lift wing to the blown high-lift wing. Conversely, a plurality of the codes only analyzed the performance of the blown high-lift cases on the geometry with the high-lift nacelles, so only those results are compared in this paper.

Additionally, as noted in the discussion of the revision 4.1 geometry in the unpowered cruise wing discussion above, a premature tip stall was noted due to an improper wing-nacelle junction. As analysis of this configuration is still ongoing, most of the CFD cases that to date have focused on the high-lift configuration have considered the high-lift wing without the wingtip cruise nacelle. The different grid generation process for the FUN3D model precluded the omission of the tip nacelle for the results in this paper.

The resulting comparison of the unblown and blown high-lift configurations are shown in Figure 8. In this case, the design-order tools were not intended to develop lift-curves, but rather, were designed to achieve a specific design lift coefficient. The X-57 design requirement for a stall speed of 58 knots calibrated airspeed resulted in a blown maximum lift coefficient requirement of 3.95. The high-lift propellers were designed to augment a wing with an unblown maximum lift coefficient of 2.6, and were further designed with a $10 \%$ high-lift augmentation margin to account for effects such as nacelle blockage, scrubbing, etc. The unblown and blown design goals are shown as a reference, and the left vs. right figures show the differences of wing-only forces vs. all high-lift propulsive forces (such as components of the high-lift thrust vector and propeller normal force included in the lift direction).

The CFD results indicate that the selected design will likely meet or exceed the high-lift performance associated with the design constraint. The wing-only forces in the left plot of Figure 8 represent the as-designed assumptions the design-order method presented earlier in this paper did not consider the propeller force components (lift component of axial thrust force and propeller normal force) in its formulation. Hence, while the unblown high-lift wing does not quite yield its maximum lift coefficient of 2.6 per the CFD, the $10 \%$ lift margin appeared to be adequate to bring the design up to the required performance level. The fact that the design method excluded direct propeller forces formed a de facto margin as well; the total force components depicted on the right of Figure 8 show that all CFD cases exceed the lift goals. The most pessimistic CFD case is the FUN3D analysis; this is fully turbulent (already a pessimistic assumption), and in both cases includes the wingtip cruise nacelle, with its known junction issues at higher angles of attack. 

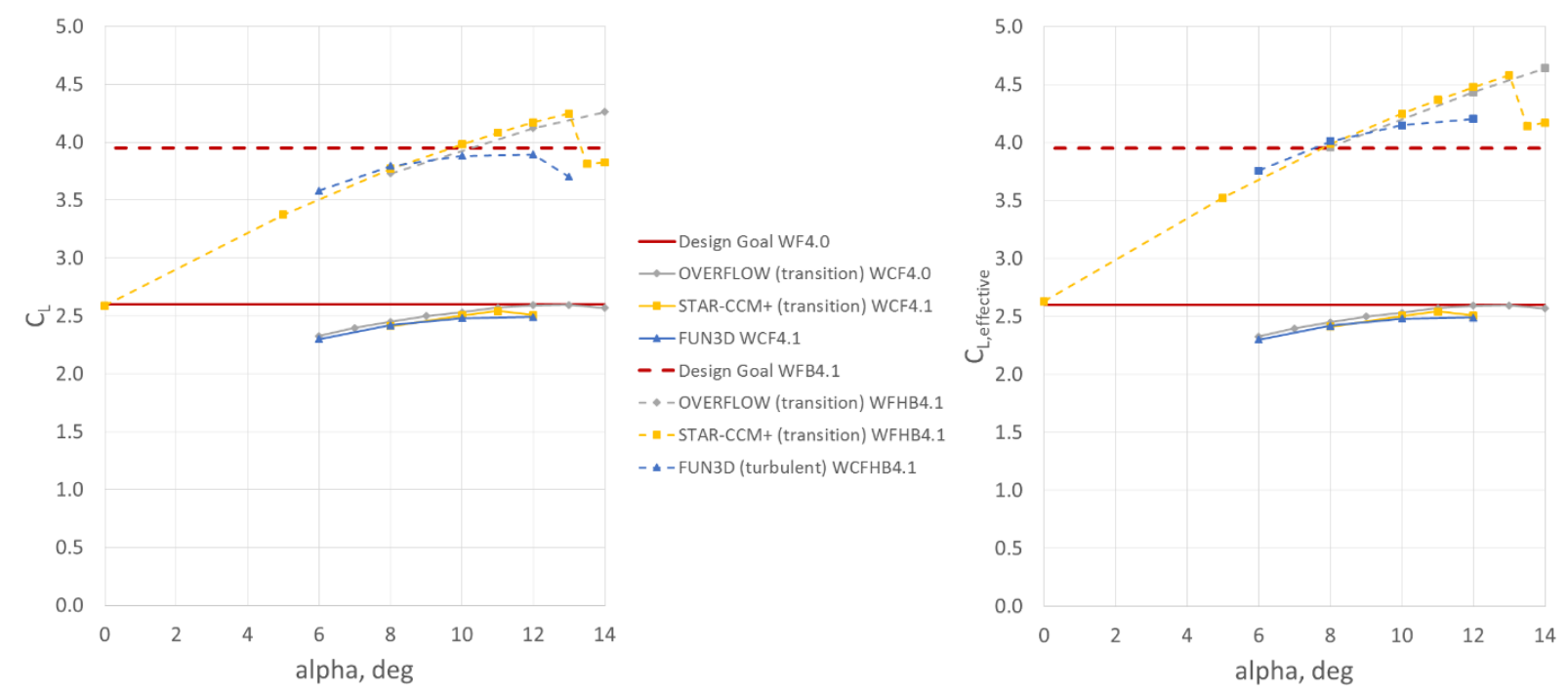

Figure 8: Comparison of Unblown and Blown High-Lift Performance - Wing Only (Left) and Wing Plus High-Lift Propeller Force Components in the Lift Direction (Right)

\section{Conclusions}

Distributed propulsion is far from a new concept, but new and improving technologies, such as electric propulsion with digital control, are rekindling interest in these concepts. Yet, while the benefits of distributed propulsion have been hypothesized and demonstrated, they have largely not yet been reduced to a series of cogent design rules and heuristics that are typically necessary to begin the aircraft design process. As such, a conceptual designer wishing to include distributed propulsion in their aircraft must find the appropriate rapid, design-order tool suite to conduct analyses and identify salient design trades.

This paper outlines the development of two distinct design-order approaches to capture the aerodynamicpropulsive benefits of different types of distributed electric propulsion technology: wingtip-mounted cruise propellers for recapture or reduction of wing vortex energy, reducing drag in cruise; and leading-edge high-lift propellers used to augment lift at low speed, enabling a smaller, more efficient wing at cruise. The development of these tools was necessitated by the goals of NASA's SCEPTOR project, and resulted in the design of the X-57 "Maxwell" distributed electric propulsion flight demonstrator. Due to the rapid nature of this design program, the project did not have the resources to conduct a traditional, wide-ranging experimental validation approach for these design-order tools. Instead, the team was able to leverage multiple, distinct, higher-fidelity computational tools, operated in parallel with the integrated design-order tools, as a means to cross-check and pseudo-validate the design results. The interim results of this ongoing pseudo-validation, presented in this paper, indicate that the performance of the X-57 should be adequate to meet program goals, and further indicates that the design-order tools used by the team yielded appropriate designs.

As part of this effort, the team enhanced multiple CFD toolchains by leveraging a common geometry engine. The use of a common, modular geometry engine enabled a pseudo-validation build-up approach, and allowed different tools to export into formats that best suited their own toolchain. Additionally, the wealth of data generated over the course of this process helped to uncover fluid-related design problems early in the design process.

\section{Acknowledgments}

This work was funded under the NASA Convergent Aeronautics Solutions (CAS) Project and Transformational Tools and Technologies (TTT) Project of the Transformative Aeronautics Concepts (TAC) Program. The authors would like to thank the entire SCEPTOR team (TAC/CAS), which includes three NASA research centers (Armstrong Flight Research Center, Glenn Research Center, and Langley Research Center) and several contractors and suppliers (Empirical Systems Aerospace, Inc., Joby Aviation, Xperimental LLC, Scaled Composites, and Electric Power Systems, TMC, and Tecnam). Additionally, the authors would like to thank the MDAO (TAC/TTT) and DELIVER teams (TAC/CAS) teams for their support of this effort. 


\section{References}

1. M. Snyder, G. Zumwalt, “Effects of Wingtip-Mounted Propellers and Turbines," Journal of Aircraft, 6(5) 392:397, 1969.

2. J. C. Patterson Jr., G. R. Bartlett, "Evaluation of Installed Performance of a Wing-Tip-Mounted Pusher Turboprop on a Semispan Wing," NASA Technical Paper TP-2739, August 1987.

3. J. D. Kummer, T. Q. Dang, "High-Lift Propulsive Airfoil with Integrated Crossflow Fan," Journal of Aircraft, 43(4) 10591068, 2006.

4. L. H. J. Smith, "Wake Ingestion Propulsion Benefit," Journal of Propulsion and Power, 9(1) 74:82, 1993.

5. J. R. Welstead, J. L. Felder, "Conceptual Design of a Single-Aisle Turboelectric Commercial Transport with Fuselage Boundary Layer Ingestion,” AIAA-2016-1027, AIAA SciTech 2016, San Diego, California, January 2016.

6. D. P. Raymer, Aircraft Design: A Conceptual Approach, Third Edition, American Institute of Aeronautics and Astronautics, Reston, Virginia, 1999.

7. J. D. Mattingly, W. H. Heiser, D. T. Pratt, Aircraft Engine Design, Second Edition, American Institute of Aeronautics and Astronautics, Reston, Virginia, 2002.

8. N. K. Borer, M. D. Patterson, J. K. Viken, M. D. Moore, S. Clarke, M. Redifer, R. Christie, A. Stoll, A. Dubois, J. Bevirt, A. Gibson, T. Foster, P. Osterkamp, "Design and Performance of the NASA SCEPTOR Distributed Electric Propulsion Flight Demonstrator," AIAA-2016-3920, AIAA Aviation, Washington, D.C., June 2016.

9. L. R. Miranda, J. E. Brennan, “Aerodynamic Effects of Wingtip-Mounted Propellers and Turbines,” AIAA-86-1802, 1986.

10. N. K. Borer, M. D. Moore, A. R. Turnbull, "Tradespace Exploration of Distributed Propulsors for Advanced On-Demand Mobility Concepts," AIAA-2014-2850, AIAA Aviation, Atlanta, GA, June 2014.

11. A. M. Stoll, J. Bevirt, M. D. Moore, W. J. Fredericks, N. K. Borer, "Drag Reduction Through Distributed Electric Propulsion," AIAA-2014-2851, AIAA Aviation, Atlanta, GA, June 2014.

12. M. D. Patterson, B. J. German. "Simplified Aerodynamics Models to Predict the Effects of Upstream Propellers on Wing Lift," AIAA 2015-1673, AIAA SciTech 2015, Kissimmee, Florida, January 2015.

13. A. S. Hahn, "Vehicle Sketch Pad: A Parametric Geometry Modeler for Conceptual Aircraft Design," AIAA-2010-0657, 48 ${ }^{\text {th }}$ AIAA Aerospace Sciences Meeting Including the New Horizons Forum and Aerospace Exposition, Orlando, Florida, January 2010.

14. J. Roskam, C.-T. E. Lan, Airplane Aerodynamics and Performance, Design, Analysis and Research Corporation, Lawrence, Kansas, 1997.

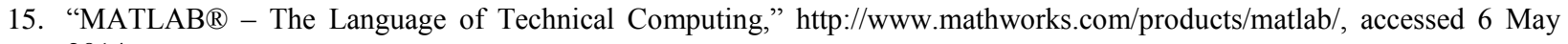
2014.

16. M. D. Patterson, B. J. German, M. D. Moore, "Performance Analysis and Design of On-Demand Electric Aircraft Concepts," AIAA-2012-5474, 12th AIAA Aviation Technology, Integration, and Operations Conference, Indianapolis, IN, 17-19 September 2012.

17. M. Drela, H. Youngren, “AVL,” http://web.mit.edu/drela/Public/web/avl/, accessed 10 May 2016.

18. M. Drela, H. Youngren, "XFOIL Subsonic Airfoil Development System," http://web.mit.edu/drela/Public/web/xfoil/, accessed 20 November 2014.

19. M. Drela, H. Youngren, "XROTOR Download Page," http://web.mit.edu/drela/Public/web/xrotor/, accessed 6 May 2014.

20. N. K. Borer, M. D. Moore, "Integrated Propeller-Wing Design Exploration for Distributed Propulsion Concepts," AIAA 2015-1672, AIAA SciTech, Kissimmee, FL, 5-9 January 2015.

21. G. Bramesfeld, A Higher Order Vortex-Lattice Method with a Force-Free Wake, Ph.D. Thesis, The Pennsylvania State University, April 2006.

22. G. Bramesfeld, M. D. Maughmer, "Relaxed-Wake Vortex-Lattice Method Using Distributed Vorticity Elements," Journal of Aircraft, 45(2):560-568, 2008.

23. M. D. Patterson, M. J. Daskilewicz, B. J. German, “Conceptual Design of Electric Aircraft with Distributed Propellers: Multidisciplinary Analysis Needs and Aerodynamic Modeling Development," AIAA-2014-0534, 52 ${ }^{\text {nd }}$ Aerospace Sciences Meeting, National Harbor, Maryland, January 2014.

24. J. Cole, M. Maughmer, G. Bramesfeld, "Aerodynamic Design Considerations for Tiltrotor Wing Extensions and Winglets," AIAA-2013-1088, 51 $1^{\text {st }}$ AIAA Aerospace Sciences Meeting including the New Horizons Forum and Aerospace Exposition, Grapevine, Texas, January 2013.

25. J. Cole, A Higher-Order Free-Wake Method for Aerodynamic Performance Prediction of Propeller-Wing Systems, Ph.D. Thesis, The Pennsylvania State University, April 2006.

26. M. D. Patterson, Conceptual Design of High-Lift Propeller Systems for Small Electric Aircraft, Ph.D. Thesis, Georgia Institute of Technology, August 2016.

27. M. D. Patterson, J. M. Derlaga, N. K. Borer, "High-Lift Propeller System Configuration Selection for NASA's SCEPTOR Distributed Electric Propulsion Flight Demonstrator,” AIAA-2016-3922, AIAA Aviation, Washington, D.C., June 2016.

28. L. Ting, C. H. Liu, G. Kleinstein, "Interference of Wing and Multipropellers", AIAA Journal, 10(7):906-914, 1972.

29. M. D. Patterson, N. K. Borer, B. J. German, "A Simple Method for High-Lift Propeller Conceptual Design," AIAA-20160770, 54 ${ }^{\text {th }}$ AIAA Aerospace Sciences Meeting, San Diego, California, January 2016.

30. "Vehicle Sketch Pad," http://www.openvsp.org/, accessed 2 December 2016.

31. J. Derlaga, N. Borer, M. Patterson, M. Moore, "Analysis of Propeller Designs and Integration Strategies During Conceptual Design via Computational Fluid Dynamics with Overset Grids,” AIAA AVIATION 2016, Washington, D.C., June 2016.

32. "NASA OVERFLOW Overset Grid CFD Flow Solver," http://overflow.larc.nasa.gov/, accessed 23 October 2015. 
33. “Chimera Grid Tools User's Manual," https://www.nas.nasa.gov/publications/software/docs/chimera/index.html, accessed 4 December 2016.

34. A. K. Kenrick, T. D. Aslam, J. M Powers, "Mapped weighted essentially non-oscillatory schemes: Achieving optimal order near critical points," Journal of Computational Physics, 207:542-567, 2005.

35. R. Tramel, R. Nichols, P. G. Buning, "Addition of Improved Shock-Capturing Schemes to OVERFLOW 2.1," AIAA-

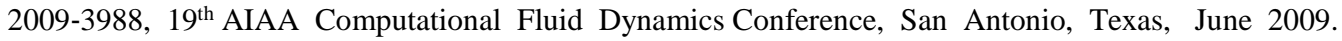

36. L Eca, M. Hoekstra, A. Hay, D. Pelletier, "A Manufactured Solution for a Two-Dimensional Steady Wall-Bounded Incompressible Turbulent Flow," International Journal of Computational Fluid Dynamics, 21(3-4):175-188, 2007.

37. P. R. Spalart, "Strategies for Turbulence Modeling and Simulations. International Journal of Heat and Fluid Flow," 21(3):252-263, 2000.

38. F. R. Menter, "Two-Equation Eddy-Viscosity Turbulence Models for Engineering Applications," AIAA Journal, 32(8):1598-1605, 1994.

39. M. L. Shur, M. K. Strelets, A. K. Traven, P. R. Spalart, "Turbulence Modeling in Rotating and Curved Channels: Assessing the Spalart-Shur Correction," AIAA Journal, 38(5):784-792, 2000.

40. F. R. Menter, R. Langtry, S. Likki, Y. Suzen, P. Huang, S. Völker, "A Correlation-Based Transition Model Using Local Variables - Part I: Model Formulation,” Journal of Turbomachinery, 128(3):413-422, 2006.

41. CD-Adapco, "STAR-CCM+," http://www.cd-adapco.com/products/star-ccm\%C2\%AE, accessed 23 October 2015.

42. P. Durbin, "On the k-3 stagnation point anomaly," International Journal of Heat and Fluid Flow, 17(1):89-90, 1996.

43. R. B. Langtry, A Correlation-Based Transition Model using Local Variables for Unstructured Parallelized CFD codes, Ph.D. Thesis, University of Stuttgart, 2006.

44. F. Stern, H. Kim, V. Patel, H. Chen, "A viscous-flow approach to the computation of propeller-hull interaction," Journal of Ship Research, 32(4):246-262, 1988.

45. R. T. Biedron, J. R. Carlson, J. M. Derlaga, P. A. Gnoffo, D. P. Hammond, W. T. Jones, B. Kleb, E. M. Lee-Rausch, E. J. Nielsen, M. A. Park, C. L. Rumsey, J. L. Thomas, W. A. Wood, "FUN3D Manual: 13.0," NASA Technical Memorandum TM-2016-219330, 2016.

46. P. R. Spalart, S. R. Allmaras, “A One-Equation Turbulence Model for Aerodynamic Flows,” La Recherche Aerospatiale, 1(1):5-21, 1994 